\title{
Ethanolaminephosphotransferase in rat lung: selectivity for endogenous and exogenous diacylglycerol
}

\author{
Hayao Ide, Joseph C. Miller and Paul A. Weinhold \\ Veterans Administration Medical Center and Deparment of Biological Chemistry, University of Michigan, \\ Ann Arbor, MI (U.S.A.)
}

(Received 7 October 1987)

Key words: Ethanolaminephosphotransferase; Phospholipid metabolism; Diacylglycerol substrate; (Rat lung)

Ethanolaminephosphotransferase (EC 2.7.8.1) activity was determined in lung microsomes using diacylglycerols generated endogenously from $\left[{ }^{14}\right.$ Clglycerol 3-phosphate and different mixtures of fatty acids. Ethanolaminephosphotransferase used endogenously generated dipalmitoylglycerol better than dioleoylglycerol. The apparent $K_{\mathrm{m}}$ and the reaction rates for four different endogenously generated mixtures were the same (16 $\mathrm{nmol} / \mathrm{mg}$ microsomal proteins). The apparent $K_{\mathrm{m}}$ values for CDP-ethanolamine were the same $(0.26 \mathrm{~mm})$ for endogenously generated dipalmitoylglycerol and dioleoylglycerol. The amount of diacylglycerol generated in microsomes was 2-3-times the apparent $K_{\mathrm{m}}$ for diacylglycerol. Dipalmitoylglycerol, supplied exogenously as a Tween 20 /phosphatidylglycerol emulsion, was nearly twice as active as dioleoylglycerol. Both dipalmitoylglycerol and dioleoylglycerol were more active as substrates when emulsions were made with phosphatidylglycerol / Tween 20 than with Tween 20 alone. The results suggest that ethanolaminephosphotransferase in lung is relatively nonselective for molecular species of diacylglycerol. In addition, the results suggest that the concentration of diacylglycerol and the physical state in which it is presented to the enzyme can affect the apparent selectivity of ethanolaminephosphotransferase for diacylglycerols.

\section{Introduction}

Phospholipid metabolism in lung tissue and isolated cells from the lung has been studied extensively because of the importance of phospholipids in the function of pulmonary surfactant, a material which lines the alveolar spaces and acts to prevent atelectasis during respiration $[1,2]$. Research has focused on the biosynthesis of 1,2-dipalmitoyl-sn-glycerol-3-phosphorylcholine (dipalmitoylphosphatidylcholine), since it is the major

Correspondence: P.A. Weinhold, Veterans Administration Medical Centre and Department of Biological Chemistry, University of Michigan, 2215 Fuller Road, Ann Arbor, MI 48105, U.S.A. surface active component in surfactant. Analysis of the molecular species of diacylglycerol in whole lung [3] and in isolated type II cells [4] revealed that disaturated diacylglycerol was present in relatively high amounts. These observations, together with results from radioisotope incorporation studies [3] and from studies on the substrate specify of cholinephosphotransferase [5-7] suggested that the de novo pathway for phosphatidylcholine synthesis was able to synthesize dipalmitoylphosphatidylcholine. Thus, the molecular species of phosphatidylcholine formed by the de novo pathway may depend on the molecular species content of the diacylglycerol pool available for the cholinephosphotransferase reaction.

Although the molecular species distribution in 
diacylglycerol and the apparent nonselective nature of cholinephosphotransferase appears to suit the requirement for the synthesis of relatively large amounts of dipalmitoylphosphatidylcholine, it presents another regulatory question. How is this process coordinated with the need to synthesize unsaturated species of phosphatidylcholine and phosphatidylethanolamine for cellular membrane formation? Phosphatidylethanolamine, for example, contains low amounts of disaturated species and a high content of polyunsaturated species, both from whole lung and isolated type II cells [4]. In order to gain insight into mechanisms which control the selection of diacylglycerols for the synthesis of phosphatidylcholine and phosphatidylethanolamine, we studied the diacylglycerol specificity for ethanolaminephosphotransferase using both endogenously generated diacylglycerol and diacylglycerol supplied exogenously as a Tween 20/phosphatidylglycerol microemulsion. The results reported in this paper indicate that ethanolamine phosphotransferase does not discriminate in the selection of diacylglycerols and can readily form phosphatidylethanolamine from dipalmitoylglycerol.

\section{Materials and Methods}

Female Sprague-Dawley rats (140-180 g) were obtained from Holtzman Co., Madison, WI. [U${ }^{14} \mathrm{C}$ Glycerol 3-phosphate $(144 \mathrm{mCi} / \mathrm{mmol})$ was purchased from New England Nucelar. CDP$\left[{ }^{14} \mathrm{C}\right]$ ethanolamine was obtained from ICN, Irvine, CA. Diisopropyl fluorophosphate was purchased from Calbiochem-Behring. All other biochemicals were from Sigma. Phosphatidate phosphohydrolase was prepared from rat liver as described by Lamb and Fallon [8].

Generation of endogenous diacylglycerol and measurement of ethanolamine phosphotransferase activity

Lung microsomes were prepared from perfused lungs as described previously [6]. Diacylglycerol within the microsomes was generated by a two-step procedure involving the sequential synthesis of phosphatidic acid from $s n$-glycerol 3-phosphate followed by hydrolysis of the phosphatidic acid to diacylglycerol by phosphatidate phosphohydro- lase. The experimental protocol was identical to that used previously for studies on cholinephosphotransferase [6]. Briefly, 6-7 mg of microsome protein were incubated for $30 \mathrm{~min}$ in $8.0 \mathrm{ml}$ of a mixture containing $\left[{ }^{14} \mathrm{C}\right]$ glycerol 3 -phosphate, $\mathrm{MgCl}_{2}$, fatty acid (complexed to albumin), KF, ATP, coenzyme A, dithiothreitol and Hepes buffer (pH 7.4). This reaction produced radioactive phosphatidic acid. The microsomes were recovered by centrifugation at $100000 \times \mathrm{g}$ for $60 \mathrm{~min}$. The microsomes were suspended in Tris buffer containing EDTA and sucrose. Aliquots of $0.1 \mathrm{ml}$ were diluted to $0.25 \mathrm{ml}$ with EDTA/diisopropyl fluorophosphate to give a solution containing $40 \mathrm{mM}$ Tris (pH 7.4), $2.0 \mathrm{mM}$ EDTA and $5 \mathrm{mM}$ diisopropyl fluorophosphate. The mixtures were incubated at $37^{\circ} \mathrm{C}$ for $20 \mathrm{~min}$, then $0.1 \mathrm{ml}$ of phosphatidate phosphohydrolase $(0.3 \mathrm{mg}$ protein) was added. This mixture was usually incubated for $20 \mathrm{~min}$. However, shorter incubations were performed in some experiments in order to generate different amounts of diacylglycerol. The ethanolaminephosphotransferase reaction was initiated by adding $150 \mu \mathrm{l}$ of a mixture which contained 0.67 $\mathrm{mM}$ Tris ( $\mathrm{pH} 8.5$ ), CDP-ethanolamine, $\mathrm{MgCl}_{2}$ and dithiothreitol so that the final concentrations were $1.0 \mathrm{mM}$ CDP-ethanolamine, $10 \mathrm{mM} \mathrm{MgCl}_{2}$ and $10 \mathrm{mM}$ dithiothreitol. Blank reactions were processed the same, except that CDP-ethanolamine was not added. The reaction was stopped by the addition of $3.0 \mathrm{ml}$ of chloroform/methanol (1:1) containing $0.1 \mathrm{M} \mathrm{HCl}$. Some samples were extracted immediately after the addition of the ethanolaminephosphotransferase reaction mixture, and these served as zero-time controls. The lipids were extracted and separated by thin-layer chromatography as described previously [6]. Different species of diacylglycerol were formed by introducing different fatty acids or mixtures of fatty acids to the first reaction mixture (i.e., for the synthesis of phosphatidic acid). The distributions of radioactivity among the species of diacylglycerol was determined by the methods described by Ishidate and Weinhold [3].

Ethanolaminephosphotransferase assay with exogenous diacylglycerol

The determination of ethanolamincphosphotransferase activity with exogenously added di- 
acylglycerol was performed using diacylglycerol microemulsions prepared as described previously [5]. The reaction mixture contained $1.0 \mathrm{mM}$ diacylglycerol, $0.8 \mathrm{mM}$ phosphatidylglycerol, 0.1 $\mathrm{mg} / \mathrm{ml}$ Tween 20, $1.0 \mathrm{mM}$ CDP- $\left[{ }^{14} \mathrm{C}\right]$ ethanolamine (200-500 dpm/mmol), $5 \mathrm{mM}$ EDTA, 5 $\mathrm{mM}$ dithiothreitol, $25 \mathrm{mM}$ magnesium acetate, $100 \mathrm{mM}$ Tris- $\mathrm{HCl}$ ( $\mathrm{pH} \mathrm{8.2)}$, and $50 \mu \mathrm{g}$ microsomal protein. The phosphatidylglycerol and Tween 20 were added as part of the diacylglycerol emulsion.

The reaction was stopped by the addition of 3.0 $\mathrm{ml}$ methanol/chloroform $(2: 1, \mathrm{v} / \mathrm{v})$. Chloroform $(1.3 \mathrm{ml})$ and water $(1.3 \mathrm{ml})$ were added, and the phases were separated by centrifugation. The chloroform phase was washed twice with $2.0 \mathrm{ml}$ of methanol/water $(1: 1, \mathrm{v} / \mathrm{v})$. The lipid extract was transferred to a scintillation vial, the chloroform was evaporated and the radioactivity was determined. Analysis of the lipid extract by thin-layer chromatography on silica gel 60 plates (E.M. Merck) in the solvent system chloroform/ methanol/acetic acid/water $(50: 30: 8: 3)$ verified that the radioactivity was located in phosphatidylethanolamine.

\section{Other methods}

The quantitative determination of diacylglycerol was done according to the method of Banschbach et al. [9]. Protein was determined by the method of Lowry et al. [10] with bovine serum albumin as the standard.

\section{Results}

Formation of phosphatidylethanolamine from endogenous diacylglycerols

Phosphatidylethanolamine was formed from $\left[{ }^{14} \mathrm{C}\right]$ diacylglycerol generated endogenously from $\left[{ }^{14} \mathrm{C}\right]$ glycerol 3-phosphate and either palmitic acid or oleic acid. The reaction was linear with time for two $\min$ (Fig. 1). The rate of reaction with dipalmitoylglycerol was higher than that with dioleoylglycerol. Analysis of the molecular species of the radioactive diacylglycerol generated from palmitate or oleate indicated that $80-90 \%$ of the $\left[{ }^{14} \mathrm{C}\right]$ diacylglycerol was the dipalmitoyl or dioleoyl species.

The relationship between the formation of phosphatidylethanolamine from endogenously

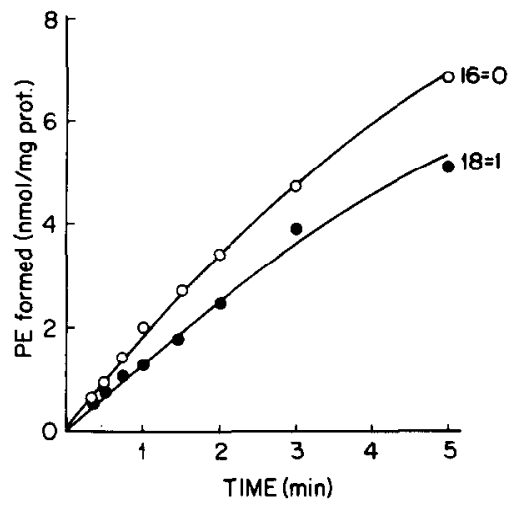

Fig. 1. The conversion of endogenously generated $\left[{ }^{14} \mathrm{C}\right] \mathrm{di}$ acylglycerol to phosphatidylethanolamine (PE) by ethanolaminephosphotransferase. Lung microsomes were incubated in the phosphatidic acid-generating system which contained $\left[{ }^{14} \mathrm{C}\right.$ glycerol 3-phosphate and either oleic acid or palmitic acid ( $0.25 \mathrm{mM}$ complexed to $6.7 \mathrm{mg}$ of albumin). After the incubation, microsomes were reisolated and suspended in $1.0 \mathrm{ml}$ of 50 $\mathrm{mM}$ Tris $/ 0.25 \mathrm{M}$ sucrose $/ 0.1 \mathrm{mM}$ EDTA (pH 7.4). Aliquots $(0.1 \mathrm{ml})$ of this mixture were distributed into tubes and treated with phosphatidic acid phosphatase for $20 \mathrm{~min}$. The ethanolaminephosphotransferase reaction was started by adding 150 $\mu 1$ of CDP-ethanolamine, $\mathrm{MgCl}_{2}$ and dithiothreitol in $0.67 \mathrm{M}$ Tris ( $\mathrm{pH} 8.5$ ), so that the final concentrations were $1.0 \mathrm{mM}$ CDP-ethanolamine, $10 \mathrm{mM} \mathrm{MgCl} 2$ and $10 \mathrm{mM}$ dithiothreitol. The reaction was stopped by the addition of $3 \mathrm{ml}$ of chloroform/methanol $(1: 1)$. The lipid was extracted and phosphatidylethanolamine was isolated by thin-layer chromatography. The concentration of diacylglycerol was determined in separate aliquots of the microsome suspension to be 32 $\mathrm{nmol} / \mathrm{mg}$ protein from oleic acid and $38 \mathrm{nmol} / \mathrm{mg}$ protein from palmitic acid. $O$, Diacylglycerol generated from palmitic acid; $\bullet$ diacylglycerol generated from oleic acid.

generated diacylglycerol and the concentration of CDP-ethanolamine is shown in Fig. 2. The apparent $K_{\mathrm{m}}$ for CDP-ethanolamine, calculated from double-reciprocal plots, was $0.27 \mathrm{mM}$ for dipalmitoylglycerol and $0.26 \mathrm{mM}$ for dioleoylglycerol.

The concentration of diacylglycerol in microsomes was varied by changing the reaction time with phosphatidate phosphohydrolase during the preparation of $\left[{ }^{14} \mathrm{C}\right]$ diacylglycerols. This enabled us to estimate the apparent $K_{\mathrm{m}}$ for endogenous diacylglycerols. Four different types of diacylglycerol were generated using different mixtures of fatty acids in the acylation of $\left[{ }^{14} \mathrm{C}\right]$ glycerol 3-phosphate. The apparent $K_{\mathrm{m}}$ values for the four different diacylglycerols were the same (16 $\mathrm{nmol} / \mathrm{mg}$ protein) (Fig. 3). The maximal velocities 


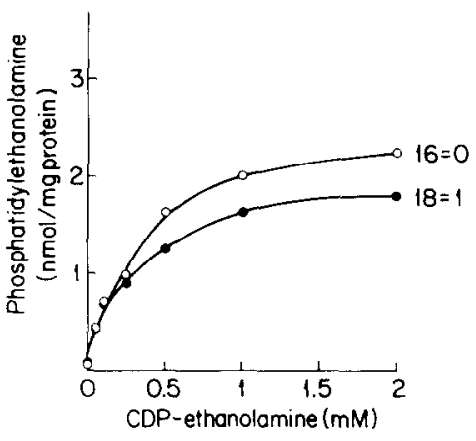

Fig. 2. The conversion of endogenously generated $\left[{ }^{14} \mathrm{C}\right] \mathrm{di}-$ acylglycerol to phosphatidylethanolamine at different CDP. ethanolamine concentrations. Diacylglycerol was generated as described in Fig. 1. The reaction was started as described in Fig. 1 using increasing concentrations of CDP-ethanolamine. The reactions were stopped after $2 \mathrm{~min}$. The diacylglycerol concentration with palmitate was $41 \mathrm{nmol} / \mathrm{mg}$ protein and with oleate was $36 \mathrm{nmol} / \mathrm{mg}$ protein. $\bullet$, Diacylglycerol from oleate; $\bigcirc$, diacylglycerol from palmitate.

calculated from Fig. 3 were 3.5, 4.8, 5.5 and 3.8 $\mathrm{nmol} / \mathrm{min}$ per mg protein for diacylglycerols generated from palmitate, palmitate/oleate, palmi-

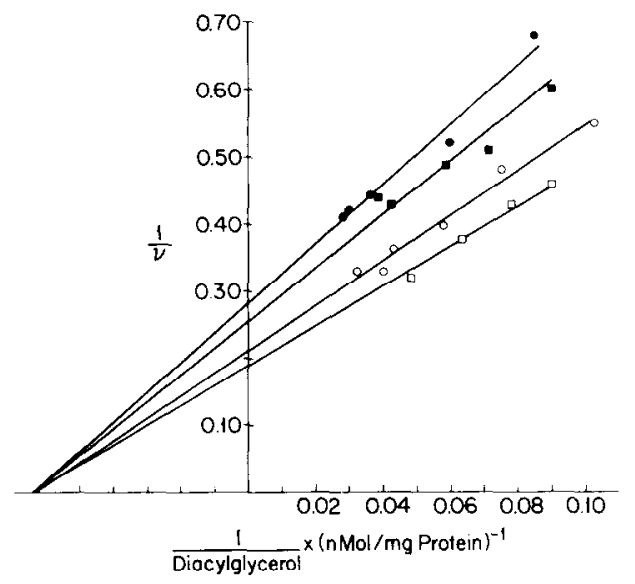

Fig. 3. Ethanolaminephosphotransferase activity with different concentrations of diacylglycerol. Diacylglycerol was generated as described in Fig. 1 using different fatty acids or mixtures of fatty acids (mixed in equal molar amounts). The total concentration of fatty acid was the same in all cascs. The amount of diacylglycerol was varied by changing the length of incubations with phosphatidic acid phosphatase. Ethanolaminephosphotransferase reaction time was $2 \mathrm{~min}$. $\bullet$, diacylglycerol generated from palmitic acid; $\bigcirc$, diacylglycerol from palmitic acid plus oleic acid; $\square$, diacylglycerol from palmitic acid plus linoleic aicd; $\mathbf{\square}$, diacylglycerol from palmitic acid plus arachidonic acid.

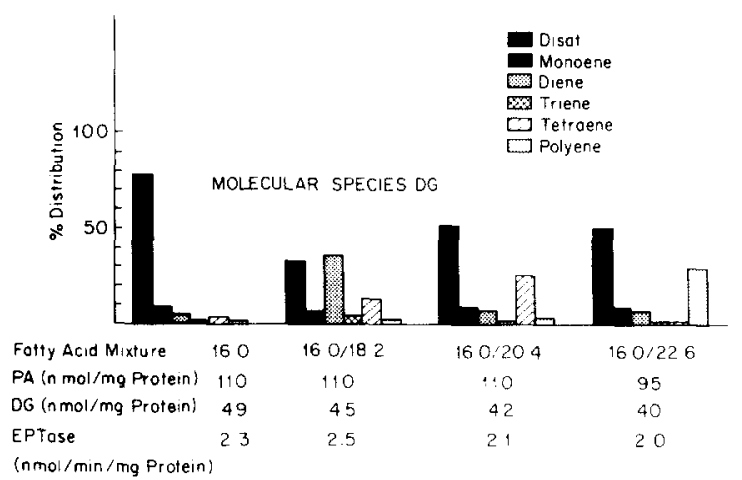

Fig. 4. Ethanolaminephosphotransferase activity (EPTase) with endogenously generated diacylglycerols which have different molecular species composition. Microsomes were incubated with fatty acid mixtures as indicated according to the protocol described in Fig. 1. After the first incubation, samples were removed and the phosphatidic acid (PA) concentrations were determined. These were calculated from the total radioactivity in phosphatidic acid divided by the specific activity of the glycerol 3-phosphate. The microsomes were then treated with phosphatidic acid phosphatase after which samples were removed for diacylglycerol (DG) analysis (both total amount and molecular species compositions). CDP-ethanolamine was added to other aliquots of the microsomal suspension and the formation of phosphatidylethanolamine was measured over a 2 min reaction.

tate/linoleate and palmitate/arachidonate, respectively.

The specificity towards endogenously generated diacylglycerols was examined further by preparing diacylglycerols which contained increasing amounts of unsaturated fatty acids. Diacylglycerols were produced with incubations containing palmitic acid, palmitic acid plus linoleic acid, palmitic acid plus arachidonic acid and palmitic acid plus docosahexaenoic acid. The mixture of fatty acids containcd equal molar amounts of each fatty acid. Separate incubations were used to determine the content of phosphatidic acid after the initial acylation, the diacylglycerol content after phosphatidic acid hydrolysis, and the rate of phosphatidylethanolamine formation. The measured rate of phosphatidylethanolamine formation was the same for all four diacylglycerol mixtures, even though the molecular species composition was different (Fig. 4).

Formation of phosphatidylethanolamine from exogenous diacylglycerol

We reported previously that diacylglycerol 


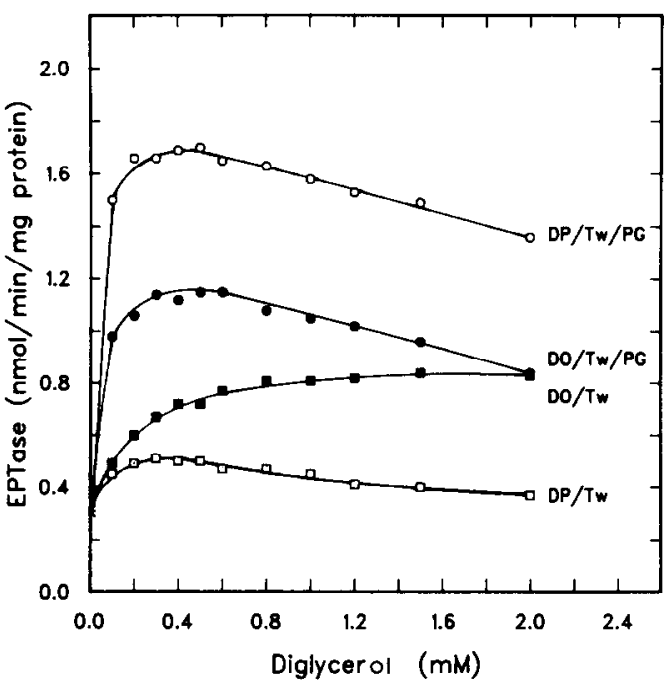

Fig. 5. Ethanolaminephosphotransferase activity in isolated microsomes using diacylglycerol added to the reaction as microemulsions. The microemulsions were formed either with phosphatidylglycerol/Tween 20 (Tw/PG) or Tween 20 (Tw) only, as described in Materials and Methods.

sonicated together with phosphatidylglycerol and Tween 20 formed microemulsions which were several-fold better substrates for cholinephosphotransferase than the usual diacylglycerol/ Tween 20 preparations [5]. We used the same methods for the preparation of diacylglycerol substrate for use in the measurement of ethanolaminephosphotransferase in lung microsomes.

Dipalmitoylglycerol prepared with Tween 20 and phosphatidylglycerol was a very effective substrate for ethanolaminephosphotransferase (Fig. 5). Dipalmitoylglycerol prepared with Tween 20 only was a poor substrate. Dioleoylglycerol prepared with Tween 20 and phosphatidylglycerol was also a better substrate than dioleoylglycerol prepared with Tween 20 . Interestingly, dipalmitoylglyccrol was apparently a better substrate than dioleoylglycerol when it was emulsified with phosphatidylglycerol. These results demonstrate that the apparent substrate specificity for ethanolaminephosphotransferase is dependent on the methods used to present the diacylglycerol to the membrane-bound enzyme system. This type of result was observed previously for cholinephosphotransferase [5].

\section{Discussion}

The results with endogenously generated diacylglycerols indicate that ethanolaminephosphotransferase is relatively nonselective for molecular species of diacylglycerols. The apparent $K_{\mathrm{m}}$ values for different mixtures of diacylglycerol molecular species were similar, as was the measured rate of the reaction. This result agrees with previous studies with liver preparations using Tween 20 emulsions of diacylglycerols [11-13]. On the other hand, others have reported that ethanolaminephosphotransferase preferentially utilizes hexenoic diacylglycerols, particularly when the diacylglycerols were contained in the membrane [14-17]. Our studies differed from these in that we employed higher concentrations of endogenous diacylglycerols. Thus, some of the reported differences between exogenously supplied diacylglycerols and endogenous diacylglycerol may be due to differences in the concentration of diacylglycerol and not necessarily to the physical form of the diacylglycerol. Holub [18] presented evidence that the selectivity of cholinephosphotransferase for certain molecular species of diacylglycerol was a function of the diacylglycerol concentration.

Both the results using endogenously generated diacylglycerols and diacylglycerols supplied exogenously as Tween 20 phosphatidylglycerol emulsions indicated that dipalmitoylglycerol was a better substrate than dioleoylglycerol. Our previous studies on the diacylglycerol specificity for cholinephosphotransferase $[5,6]$ showed that, although dipalmitoylglycerol was a very active substrate, dioleoylglycerol was an equal to better substrate. Thus, the results with ethanolaminephosphotransferase were unexpected, and may reflect a more pronounced preference for diacylglycerols with saturated fatty acids at the $s n-1$ position.

Rustow and Kunze [7] reported that ethanolaminephosphotransferase used exogenously supplied dipalmitoylglycerol, but did not use endogenously supplied dipalmitoylglycerol. Similarly, Post et al. [19] could not detect the formation of dipalmitoylphosphatidylethanolamine from endogenous dipalmitoylglycerol in isolated type II cells. Both Rustow and Kunze [7] and Post et al. [19] measured ethanolaminephosphotransferase 
activity with endogenous diacylglycerol concentrations present in freshly isolated microsomes. These levels are considerably lower than those in our system, since we generated diacylglycerol prior to measuring ethanolaminephosphotransferase activities. Thus, we employed diacylglycerol concentrations 2-3-times the apparent $K_{\mathrm{m}}$ values. Under these conditions, ethanolaminephosphotransferase does not appear to discriminate against dipalmitoylglycerol. These results support the conclusion made by Rustow and Kuntz [7] that the lack of formation of dipalmitoylphosphatidylethanolamine in vivo is not because of a discrimination of ethanolaminephosphotransferase against dipalmitoylglycerol, but instead, it is the availability of dipalmitoylglycerol which directs the synthesis. This envisions separate pools of diacylglyccrol for cholinephosphotransferase and ethanolaminephosphotransferase. If this is, indeed, the case, the generation of relatively high amounts of diacylglycerol may effectively swamp out any pool effects.

Another plausible mechanism for the control of the flow of diacylglycerols to phosphatidylcholine and phosphatidylethanolamine is suggested by the results from the experiments which used diacylglycerol/Tween 20/phosphatidylglycerol emulsions as exogenous substrates. The ability of ethanolaminephosphotransferase to use different diacylglycerols depended upon the way that diacylglycerols were presented to the enzyme system. When dioleoylglycerol and dipalmitoylglycerol are prepared with Tween 20, ethanolaminephosphotransferase utilized the dioleoylglycerol better than dipalmitoylglycerol. However, when these substrates were prepared with Tween 20 and phosphatidylglycerol, the dipalmitoylglycerol appeared to be a better substrate. Thus, the phospholipid environment at the ethanolaminephosphotransferase site within the membrane may also influence the diacylglycerol selectivity of the enzyme.

\section{Acknowledgements}

This work was supported by the Veterans Administration Research Service and by Grants HD 02871 and HD 21992 from the National Institutes of Health.

\section{References}

1 Pattle, R.E. (1958) Proc. R. Soc. Lond. Ser. B 148, 217-240.

2 King, R.J. (1974) Fed. Proc. 33, 2238-2245.

3 Ishidati, K. and Weinhold, P.A. (1981) Biochim. Biophys. Acta 664, 133-147.

4 Crecelius, C.A. and Longmore, W.J. (1984) Biochim. Biophys. Acta 795, 247-256.

5 Miller, J.C. and Weinhold, P.A. (1981) J. Biol. Chem. 256, 12662-12665.

6 Ide, H. and Weinhold, P.A. (1982) J. Biol. Chem. 257, 14926-14931.

7 Rustow, B. and Kunze, D. (1984) Biochim. Biophys. Acta 793, 372-378.

8 Lamb, R.G. and Fallon, H.J. (1974) Biochim. Biophys. Acta 348, 166-178.

9 Banschbach, M.W., Geison, R.L. and O'Brien, J.F. (1974) Anal, Biochem. 49, 617-627.

10 Lowry, O.H., Rosebrough, N.J., Farr, A.L. and Randall, R.J. (1951) J. Biol. Chem. 193, 265-275.

11 Mudd, J.B., Van Golde, L.M.G. and Van Deenen, L.L.M. (1969) Biochim. Biophys. Acta 176, 647-556.

12 Dekruyff, B., Van Golde, L.M.G. and Van Deenen, L.L.M. (1970) Biochim. Biophys. Acta 210, 425-435.

13 Kanoh, H. (1970) Biochim. Biophys. Acta 218, 249-258

14 Young, D.L. and Lynen, F. (1969) J. Biol. Chem. 244. 377-383.

15 Kanoh, H. and Ohno, K. (1975) Biochim. Biophys. Acta 380, 199-207.

16 Roberti, R., Binaglia, L. and Porcellati, G. (1980) J. Lipid Res. 21, 449-454.

17 Holub, B.J. (1978) J. Biol. Chem. 253, 691-696.

18 Holub, B.J. (1977) Can. J. Biochem. 55, 700-705.

19 Post, M., Schuurmans, E.J.M., Batenburg, J.J. and Van Golde, L.M.G. (1983) Biochim. Biophys. Acta 750, 68-77. 